\title{
Comparison of optical spectra recorded during DPF-I000U plasma experiments with gas-puffing
}

Dobromil R. Zaloga, Elzbieta Skladnik-Sadowska, Monika Kubkowska, Marina S. Ladygina, Karol Malinowski, Roch Kwiatkowski, Marek J. Sadowski, Marian Paduch, Ewa Zielinska, Vadym A. Makhlaj

\begin{abstract}
The results are presented of the optical spectra measurements for free plasma streams generated with the use of the modified DPF-1000U machine. This facility was recently equipped with a gas injection system (the so-called gas-puff) placed on the symmetry axis behind the central opening in the inner electrode. The DPF-1000U experimental chamber was filled up with pure deuterium at the initial pressure of 1.6 or 2.4 mbar. Additionally, when the use was made of the gas-puff system about $1 \mathrm{~cm}^{3}$ of pure deuterium was injected at the pressure of 2 bars. The gas injection was initiated 1.5 or $2 \mathrm{~ms}$ before the triggering of the main discharge. The investigated plasma discharges were powered from a condenser bank charged initially to $23 \mathrm{kV}$ (corresponding to the energy of $352 \mathrm{~kJ}$ ), and the maximum discharge current amounted to about 1.8 MA. In order to investigate properties of a dense plasma column formed during DPF-1000U discharges the use was made of the optical emission spectroscopy. The optical spectra were recorded along the line of sight perpendicular to the vacuum chamber, using a Mechelle ${ }^{\circledR} 900$ spectrometer. The recent analysis of all the recorded spectra made it possible to compare the temporal changes in the electron density of a freely propagating plasma stream for discharges without and with the gas-puffing. Using this data an appropriate mode of operation of the DPF-1000U facility could be determined.
\end{abstract}

Key words: electron density $\bullet$ gas-puff $\bullet$ optical spectrum $\bullet$ plasma focus $\bullet$ plasma stream

D. R. Zaloga ${ }^{\bowtie}$, E. Skladnik-Sadowska, R. Kwiatkowski, K. Malinowski

National Centre for Nuclear Research (NCBJ),

7 Andrzeja Soltana Str., 05-400 Otwock/Swierk, Poland,

Tel.: +48 22273 1417, Fax: +48 22779 3481,

E-mail: dobromil.zaloga@ncbj.gov.pl

M. Kubkowska, M. Paduch, E. Zielinska

Institute of Plasma Physics and Laser Microfusion

(IPPLM),

23 Hery Str., 01-497 Warsaw, Poland

M. S. Ladygina, V. A. Makhlaj

Institute of Plasma Physics, NSC KIPT,

61-108 Kharkov, Ukraine

M. J. Sadowski

National Centre for Nuclear Research (NCBJ),

7 Andrzeja Soltana Str., 05-400 Otwock/Swierk, Poland and Institute of Plasma Physics and Laser Microfusion (IPPLM),

23 Hery Str., 01-497 Warsaw, Poland

Received: 12 June 2014

Accepted: 14 January 2015

\section{Introduction}

Investigation of the optical spectra emitted from plasma streams is of primary importance for determination of the composition and the dynamics of such plasma objects. The optical emission spectroscopy (OES) has already been used for the investigation of intense pulsed plasma-ion streams generated with the use of the 1 MJ plasma focus facility [1-4], but recent modifications of this machine, and particularly the installation of the gas-puffing, have made it necessary to perform anew the detailed spectroscopic measurements. In this note the most important results are presented of experimental studies performed with the use of the modified DPF-1000U facility without and with the gas-puffing.

\section{Experimental setup}

The DPF-1000U facility is equipped with two $460 \mathrm{~mm}$ long coaxial electrodes. The inner electrode has the form of a thick copper tube $230 \mathrm{~mm}$ in diameter, and its front plate has an axial hole $50 \mathrm{~mm}$ 
in diameter, behind which the fast-acting gas valve outlet nozzle is located. The outer electrode is $400 \mathrm{~mm}$ in diameter and consists of twelve stainless-steel tubes $80 \mathrm{~mm}$ in diameter, which are symmetrically distributed around the axis [5].

During the reported experiments the DPF-1000U chamber was filled up with pure deuterium under a pressure of $p_{o}=1.6$ or 2.4 mbar. Some discharges were performed without gas-puffing, but in the cases when the gas-puffing was applied about $1 \mathrm{~cm}^{3}$ of pure deuterium under a pressure of 2 bars was injected along the axis 1.5 or $2 \mathrm{~ms}$ before the discharge was triggered. It should be noted, however, that the triggering of the gas valve at the chosen instant did not cause an instantaneous injection of the whole gas amount (ca. $10^{20}$ deuterium atoms). The gas valve remained open for at least several milliseconds, and the inflowing gas formed a stream along the $\mathrm{z}$-axis practically during the whole discharge lasting several microseconds [1]. For this reason the injected gas has not been 'dissolved' in the gas filling the whole discharge chamber, but it formed a gas target for the collapsing current sheath. Therefore, an influence of the gas-puffing was noticeable, e.g., on laser interferometric images as well as on X-ray and neutron emission characteristics [6].

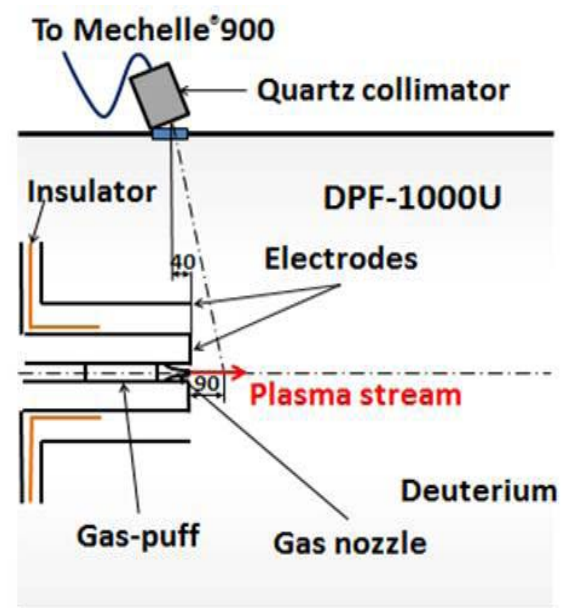

Fig. 1. The scheme of the DPF-1000U facility and the experimental arrangement for optical emission spectroscopy measurement.

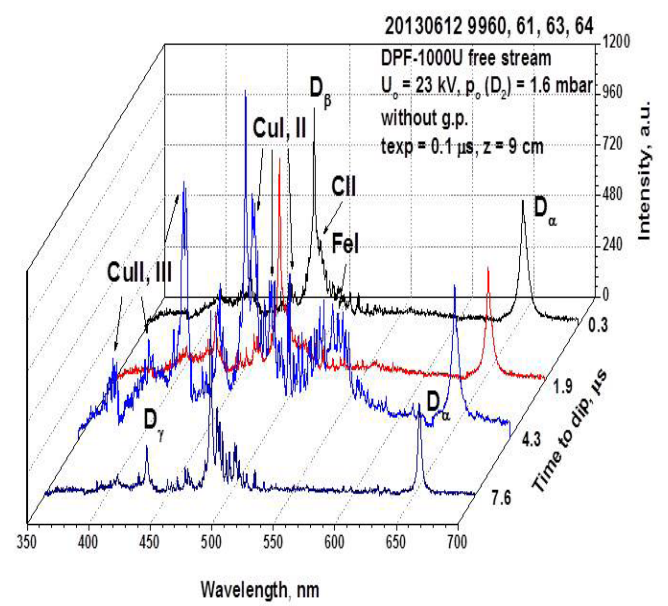

It should be added that during the reported experiments the DPF-1000U discharges were powered from a condenser bank charged to $U_{o}=23 \mathrm{kV}$, which corresponded to $W_{0}=352 \mathrm{~kJ}$, and the maximum discharge current amounted to $1.8 \mathrm{MA}$ in about $5 \mu \mathrm{s}[1]$.

As mentioned above, in order to investigate the generated plasma-ion streams the optical emission spectra were registered in the visible range. Measurements were performed by means of a Mechelle ${ }^{\circledR} 900$ spectrometer coupled through an optical fibre cable with a collimator situated behind an optical window. Those optical spectra were recorded along a line of sight almost perpendicular to the z-axis, at a distance of $9 \mathrm{~cm}$ from the electrode ends, where the target was located during the previous experiments $[4,5]$. The experimental arrangement for this experiment is presented in Fig. 1.

\section{Experimental results}

In order to compare the optical emission spectra for DPF-1000U discharges carried out under different experimental conditions, the optical measurements were initially performed without any gas-puffing. It should be noted that in comparison with the optical spectra observed in the previous PF-1000 experiments [2-4] the spectra recorded recently in the DPF-1000U facility without the gas-puffing showed negligible differences in lines shapes and intensities. Some examples of the optical spectra recorded at different instants before and after the discharge current peculiarity (the so-called current dip) are shown in Fig. 2.

For the DPF-1000U discharges performed with the gas-puffing some important differences were observed. Examples of the optical spectra recorded at different initial pressures in the experimental chamber are presented in Figs. 3 and 4.

One can easily see that in the considered cases there appeared many impurity lines, and their intensities increased considerably in later phases of the discharge. In particular, spectral lines corresponding to $\mathrm{Cu}, \mathrm{Fe}$ and $\mathrm{C}$ elements were recorded. For a comparison, some changes in the profile of the $\mathrm{D}_{\alpha}$

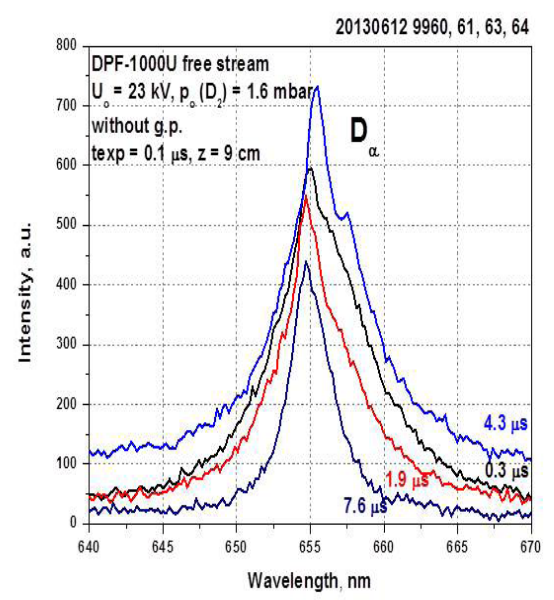

Fig. 2. Temporal changes of the optical spectra recorded for the DPF-1000U shots at $p_{o}=1.6 \mathrm{mbar}$ and $U_{o}=23 \mathrm{kV}$ (left); profiles of $D_{\alpha}$ at different instants after the current dip (right). 

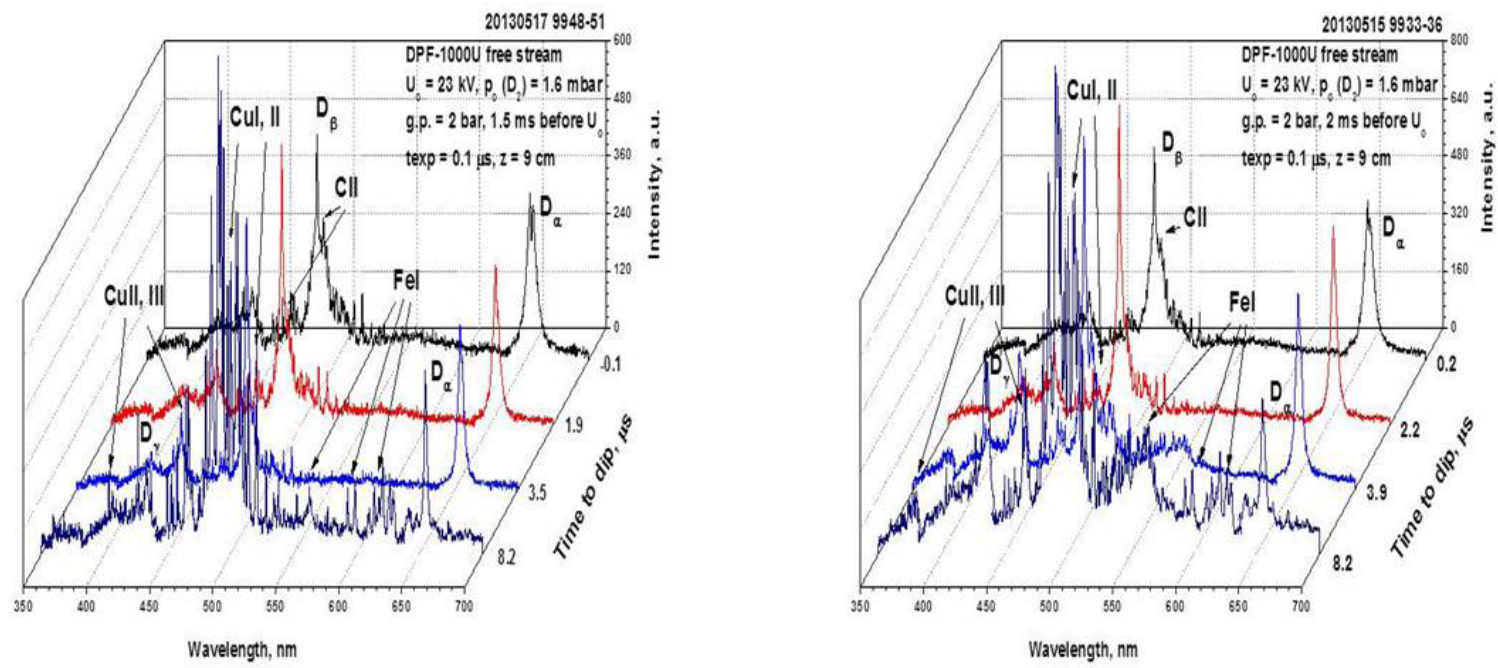

Fig. 3. Temporal changes of the optical spectra for DPF-1000U shots performed at $p_{o}=1.6 \mathrm{mbar}$ and $U_{o}=23 \mathrm{kV}$, with the gas-puffing at various instants: $1.5 \mathrm{~ms}$ (left) and $2.0 \mathrm{~ms}$ (right) before the application of the high-voltage pulse.

line, as observed in the spectra presented in Figs. 3 and 4, are shown in Figs. 5 and 6.

It should be noted that a strong absorption of this line was observed at $t_{\mathrm{dip}}=0$, i.e. when the plasma

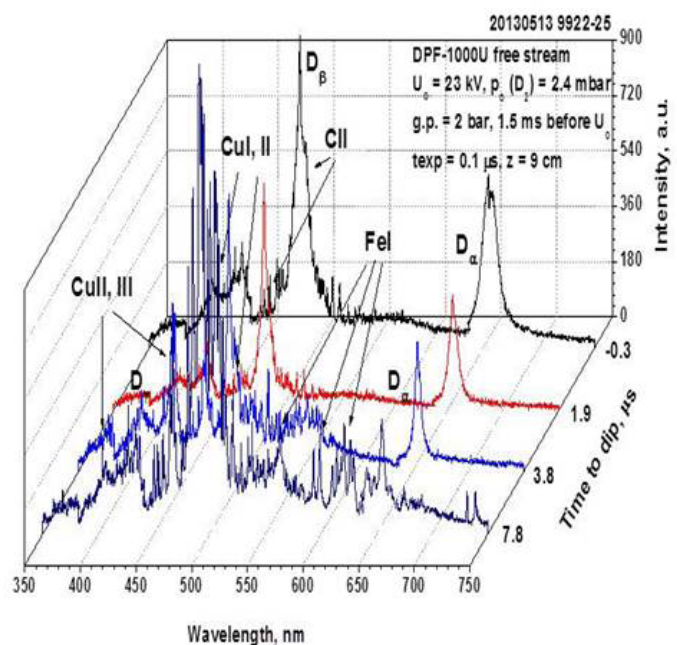

column had the most dense structure. Apart from the $\mathrm{D}_{\alpha}$ line also distinct lines of CII ions were seen, especially near $t_{\text {dip }}=0$.

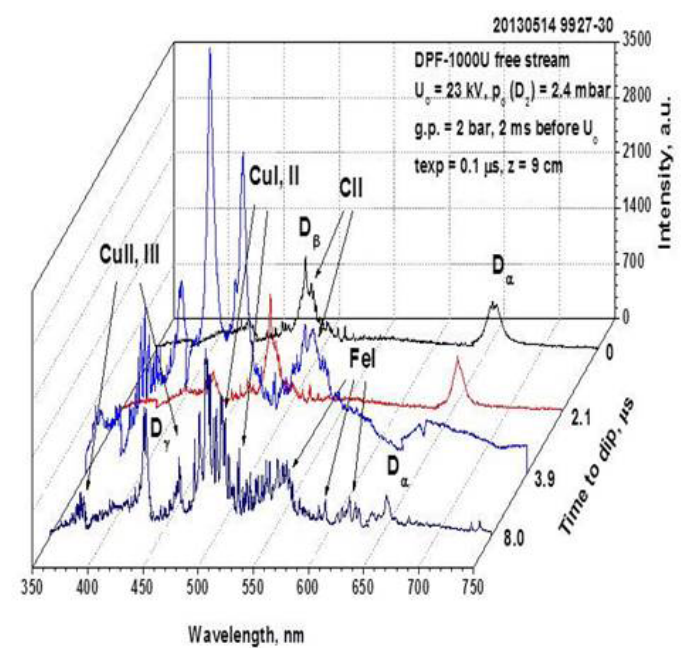

Fig. 4. Temporal changes of the optical spectra for DPF-1000U shots performed at $p_{o}=2.4$ mbar and $U_{o}=23 \mathrm{kV}$, with the gas-puffing at various instants: $1.5 \mathrm{~ms}$ (left) and $2.0 \mathrm{~ms}$ (right) before the application of the high-voltage pulse.
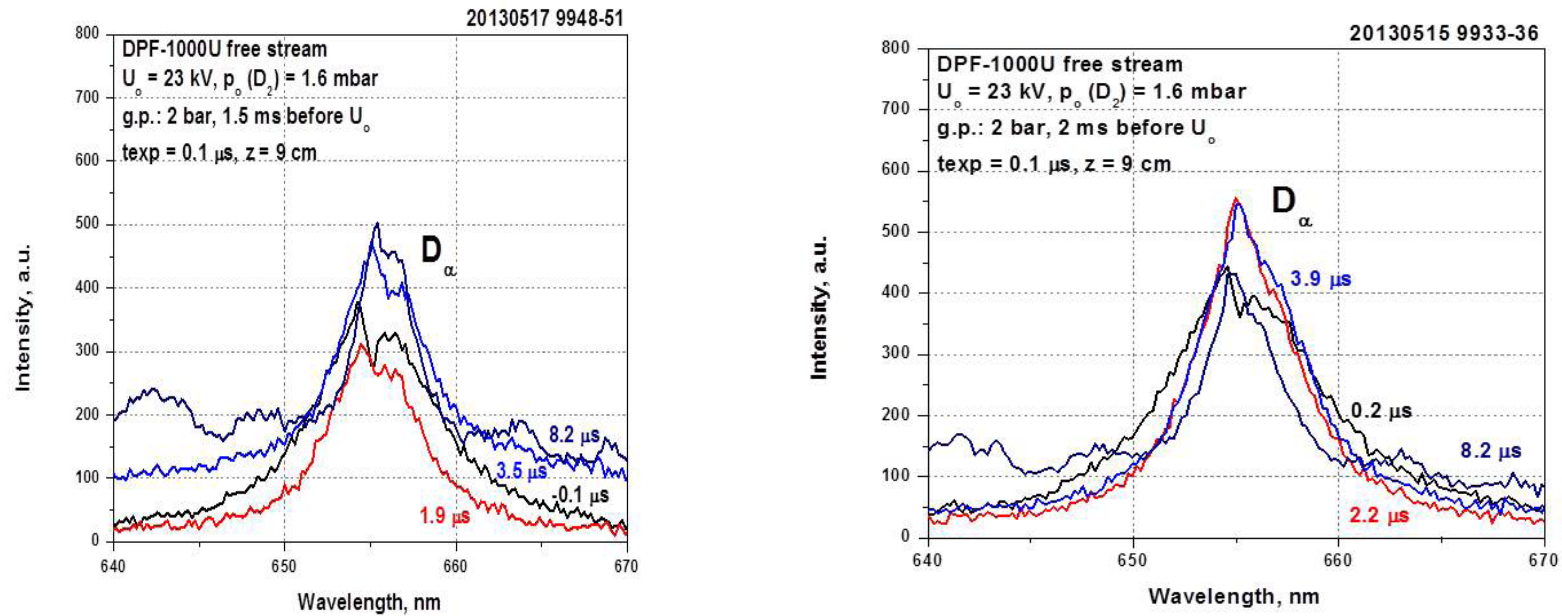

Fig. 5. Profiles of the $\mathrm{D}_{\alpha}$ line measured at $p_{o}=1.6 \mathrm{mbar}, U_{o}=23 \mathrm{kV}$ and different instants after the current dip, as recorded for DPF-1000U shots with the gas-puffing performed $1.5 \mathrm{~ms}$ (left) and $2.0 \mathrm{~ms}$ (right) before the discharge was triggered. 

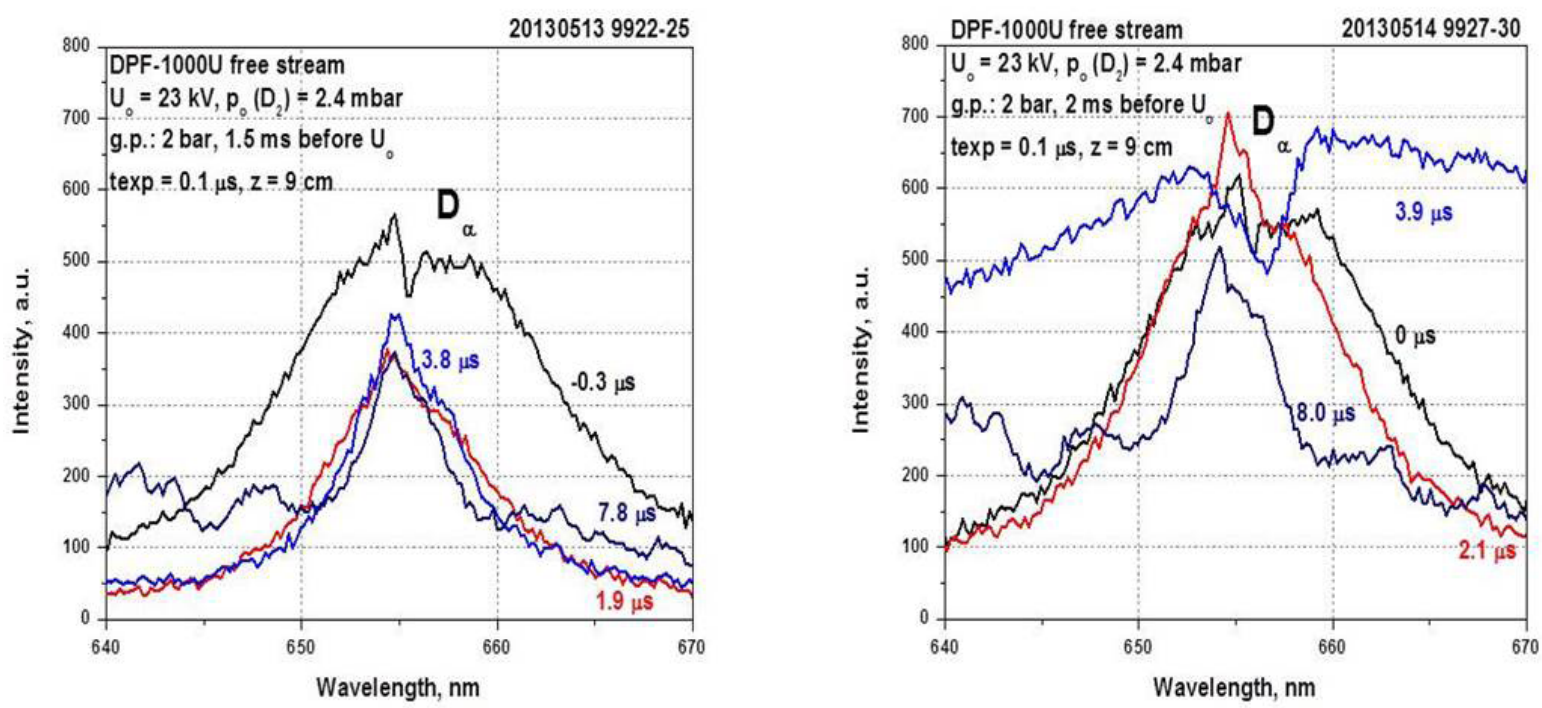

Fig. 6. Profiles of the $\mathrm{D}_{\alpha}$ line measured at $p_{o}=2.4 \mathrm{mbar}, U_{o}=23 \mathrm{kV}$ and different instants after the current dip, as recorded for DPF-1000U shots with the gas-puffing performed $1.5 \mathrm{~ms}$ (left) and $2.0 \mathrm{~ms}$ (right) before the discharge was triggered.

\section{Analysis of experimental results}

In order to determine the plasma density in DPF-1000U discharges without gas-puffing the recorded $\mathrm{D}_{\alpha}$ lines were fitted by a Lorentzian profile. An example is presented in Fig. 7.

Analogous fitting was performed also for the $D_{\alpha}$ line recorded during DPF-1000U discharges carried out with the gas-puffing. On the basis of those fits and taking into account the full width at the half maximum (FWHM) it was possible to estimate the corresponding plasma electron density values $n_{e}$. The use was made of the known formula [7]:

$$
\Delta \lambda_{1 / 2}^{S}=2.5 \times 10^{-9} \alpha_{1 / 2} \cdot\left(n_{e}\right)^{2 / 3}
$$

where $\Delta \lambda_{1 / 2}$ is a Stark full width at the half maximum of the analyzed spectral line, and $\alpha_{1 / 2}$ is a fractional semi-half-width parameter taken from literature [8, 9], which depends on the plasma temperature.

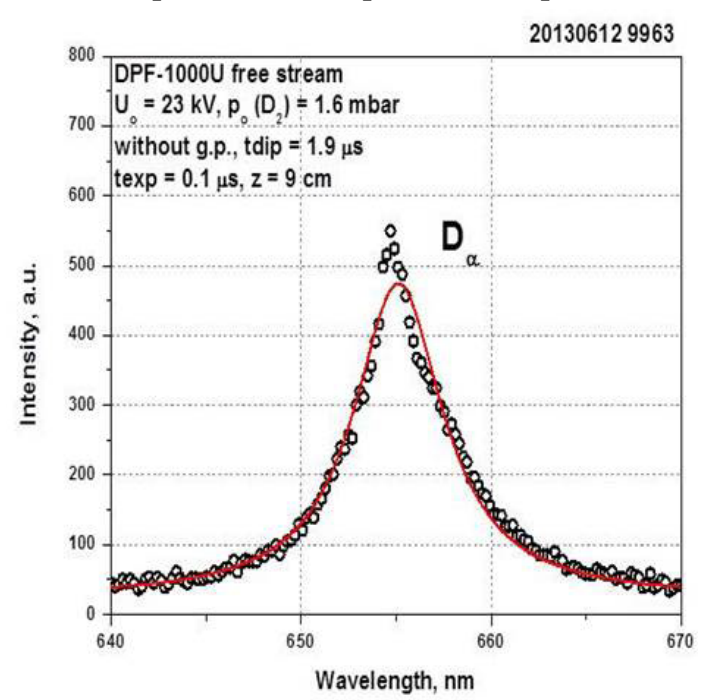

Fig. 7. Fitting of the Lorentzian profile to the $\mathrm{D}_{\alpha}$ line from the optical spectrum obtained in the DPF-1000U discharge performed at $p_{o}=1.6$ mbar, $U_{o}=23 \mathrm{kV}$, without gas-puffing, as recorded $1.9 \mu$ s after the current dip.
On the basis of the above formula and the FWHM values measured for the investigated $D_{\alpha}$ lines, which were recorded at different moments during the discharges without and with the gas-puffing, it was possible to perform corresponding computations. They were carried out for the investigated DPF-1000U discharges, under an additional assumption that the plasma temperature range was similar to that determined in earlier experiments $[10,11]$, and their results are presented in Fig. 8 .

It should here be noted that the estimates of the averaged plasma density for discharges without gas-puffing presented here do not differ considerably from results of earlier interferometric measurements of freely propagating plasma streams in the PF-1000 facility (i.e. without any target) [4].

From the diagrams presented in Fig. 8 it can also be seen that in the first phase of the DPF-1000U pinch column decay (until about $1.5 \mu \mathrm{s}$ ), application of the gas-puffing resulted in a higher electron density of the generated plasma streams, which in most cases reached values as high as $6 \times 10^{18} \mathrm{~cm}^{-3}$. In the later phase of the pinch column decay the observed changes in electron densities were not so strong and the estimated values amounted to about $(1-2) \times 10^{18} \mathrm{~cm}^{-3}$.

It should be noted that the density values obtained for deuterium discharges under the initial pressure of $p_{o}=2.4$ mbar were noticeably higher than those recorded at $p_{o}=1.6$ mbar. One should also note that these estimates refer to plasma density values on the z-axis of the pinch column, at a distance of $9 \mathrm{~cm}$ from the electrode outlets. The obtained values were of course averaged along the optical observation axis.

\section{Summary and conclusions}

The most important results of the reported study can be summarized as follows: the measurements performed by means of the optical emission spec- 
troscopy showed that for the DPF-1000U discharges performed with the gas-puffing the electron density in the pinch column (during the first instants of its decay) was considerably higher (about $6 \times 10^{18} \mathrm{~cm}^{-3}$ ) compared to values measured for discharges without gas-puffing (about $(1-2) \times 10^{18} \mathrm{~cm}^{-3}$ ).

Such a difference was particularly evident in earlier phases of the pinch decay after the current peculiarity. In later phases of the pinch decay the electron densities were comparable in both cases, i.e. with and without the gas-puffing, and their values amounted to about $(1-2) \times 10^{18} \mathrm{~cm}^{-3}$. It should be noted that estimates of the plasma electron density were performed on the basis of the $\mathrm{D}_{\alpha}$ line profiles, because the $\mathrm{D}_{\beta}$ line showed complex shapes, probably due to closely lying impurity lines (of $\mathrm{Cu}$ - and $\mathrm{Fe}$-species). Error bars were not marked in diagrams presented in Fig. 8, but under the assumption about the temperature range (see above) they were estimated to amount to about 30-50\% of the given values. Nevertheless, the measurements performed proved valuable for the determination of the discharge characteristics.
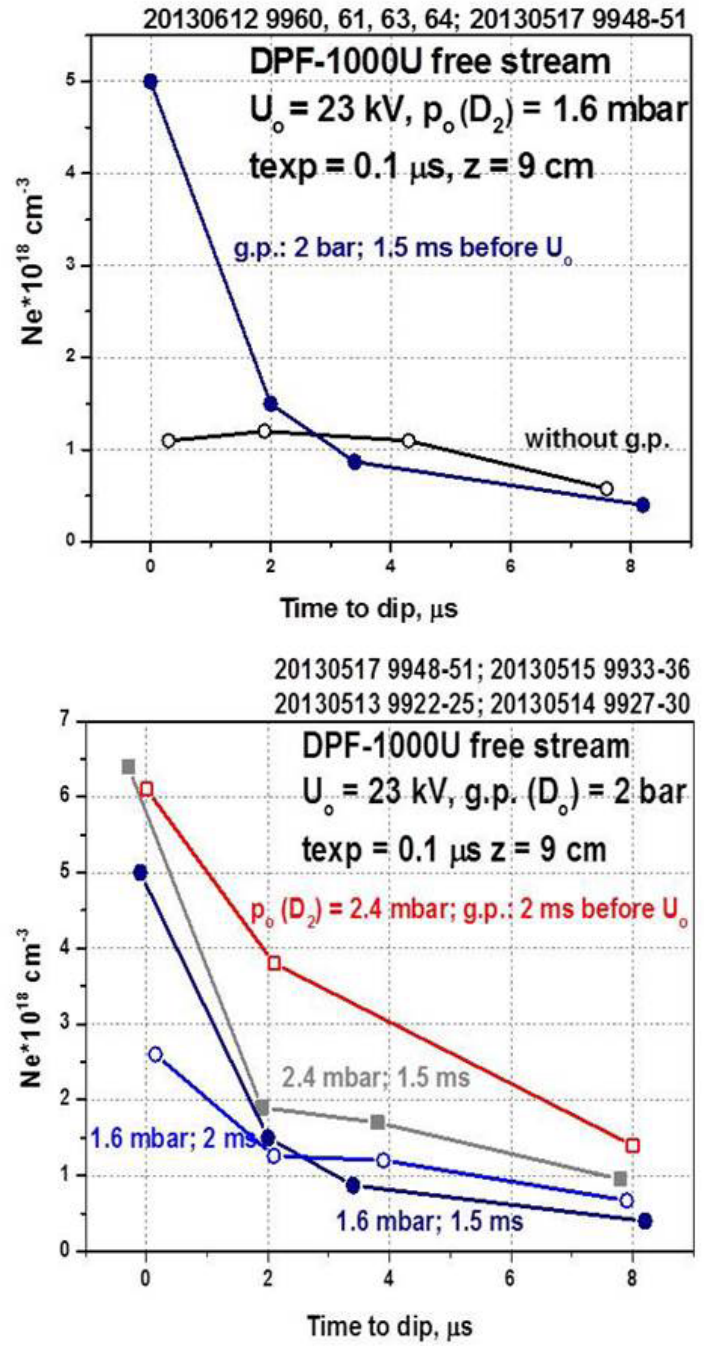

Fig. 8. Temporal changes of the plasma electron density on the $\mathrm{z}$-axis at a distance of $9 \mathrm{~cm}$ from the electrode outlets, as determined for the DPF-1000U discharges performed without and with the gas-puffing.
Acknowledgment. This work was performed within the strategic research project "Technologies supporting the development of safe nuclear power" financed by the National Centre for Research and Development (NCBR), Poland, as a research task "Research and development of techniques for the controlled thermonuclear fusion" under the contract no. SP/J/2/143234. Work was performed at NCBJ in Otwock-Swierk and at IPPLM in Warsaw, Poland. This work was partly supported by the IAEA CRP RC-16954 and RC-16956 grants as well by the Polish Ministry of Science and Higher Education within the framework of the financial resources in the year 2014 allocated for the realization of the international co-financed projects.

\section{References}

1. Scholz, M., Drozdowicz, K., Karpinski, L., Krauz, V., Kubes, P., Paduch, M., Pisarczyk, T., Sadowski, M. J., Kurowski, A., Igielski, A., Dankowski, J., Woznicka, U., Janik, W., Czaus, K., Malinowski, K., Kwiatkowski, R., Skladnik-Sadowska, E., \& Zebrowski, J. (2013). Status of Plasma-Focus research in Poland. In Proceedings of the International Conference PLASMA-2013, 2-6 September 2013 (T10). Warsaw, Poland.

2. Skladnik-Sadowska, E., Kwiatkowski, R., Malinowski, K., Sadowski, M. J., Kubkowska, M., Paduch, M., Scholz, M., \& Zielinska, E. (2012). Optical emission spectroscopy of pulsed plasma streams emitted from a modified PF-1000 facility. Problems of Atomic Science and Technology, Seria: Plasma Phys., 82(6), 246-248.

3. Skladnik-Sadowska, E., Kwiatkowski, R., Malinowski, K., Sadowski, M. J., Żebrowski, J., Kubkowska, M., Paduch, M., Scholz, M., Gribkov, V. A., Garkusha, I. E., Ladygina, M. S., \& Marchenko, A. K. (2013). Optical emission spectroscopy of free-propagating plasma streams and plasma produced during their interactions with solid targets. Problems of Atomic Science and Technology, Seria: Plasma Phys., 83(1), 279-283.

4. Kubkowska, M., Skladnik-Sadowska, E., Kwiatkowski, R., Malinowski, K., Kowalska-Strzęciwilk, E., Paduch, M., Sadowski, M. J., Pisarczyk, T., Chodukowski, T., Kalinowska, Z., Zielinska, E., \& Scholz, M. (2014). Investigation of interactions of intense plasma streams with tungsten and CFC targets in the PF-1000 facility. Phys. Scripta, T161, 014038(5pp.). DOI: 10.1088/0031-8949/2014/T161/014038.

5. Jakubowska, K., Kubkowska, M., Skladnik-Sadowska, E., Malinowski, K., Marchenko, A. K., Paduch, M., Sadowski, M. J., \& Scholz, M. (2011). Optical emission spectroscopy of plasma streams in PF-1000 experiments. Nukleonika, 56(2), 125-127.

6. Kubes, P., \& Paduch, M., Cikhardt, J., Kortanek, J., Batobolotova, B., Rezac, K., Klir, D., Kravarik, J., Surala, W., Zielinska, E., Scholz, M., Karpinski, L., \& Sadowski, M. J. (2014). Neutron production from puffing deuterium in plasma focus device. Phys. Plasmas, 21, 082706.

7. Griem, H. R. (1964). Plasma spectroscopy. New York: McGraw Hill.

8. Kepple, P., \& Griem, H. R. (1968). Improved stark profile calculations for the hydrogen lines $\mathrm{H}_{\alpha}, \mathrm{H}_{\beta}, \mathrm{H}_{\gamma}$, and $\mathrm{H}_{\delta}$. Phys. Rev., 176(1), 317-325. DOI: 10.1103/ PhysRev.173.317. 
9. Griem, H. R. (1974). Spectral line broadening by plasmas. New York: Academic Press.

10. Scholz, M., Karpinski, L., Paduch, M., Pisarczyk, T., Zielinska, E., Chodukowski, T., Sadowski, M. J., Składnik-Sadowska, E., Czaus, K., Kwiatkowski, R., Malinowski, K., Krauz, S., \& Mitrovanov, K. (2010). MJ Plasma-Focus diagnostic systems. In Proceedings of the International Conference Plasma Diagnostics, 12-16 April 2010. Pont-a-Mousson, France. (Invited).
11. Skladnik-Sadowska, E., Malinowski, K., Sadowski, M. J., Kubkowska, M., Jakubowska, K., Paduch, M., Scholz, M., Garkusha, I. E., Ladygina, M. S., \& Tereshin, V. I. (2010). Optical spectroscopy of free-propagating plasma and its interaction with tungsten targets in PF-1000 facility. Contr. Plasma Phys., 51(2/3), 288-292. DOI: 10.1002/ctpp.201000046. 